\title{
Halley Irrational-Homotopy Analysis Method
}

\author{
Aml Melad Shloof and Mohd Salmi Noorani \\ School of mathematical sciences Faculty of science and technology \\ University kebangsaan Malaysia \\ E-mail: Amlshalouf@yahoo.com, msn@ukm.my
}

\begin{abstract}
In this paper we investigate Halley irrational-homotopy analysis method as a new technique to find approximate solution of nonlinear equations, where complex and real roots are founded and in many cases we have two solutions. The Halley's irrational formula developed to determine the auxiliary (arbitrary) parameter in HAM. Furthermore, we also discuss in detail the order of convergence of this method. In order to validated and substantiate the performance of our contributed scheme, we give some examples.
\end{abstract}

Keywords: Homotopy analysis method (HAM), Complex and real roots, Convergence analysis, Auxiliary parameter $\hbar$, Halley irrational method.

\section{Introduction}

In the past six years, homotopy analysis method (HAM) has become a famous and widely applied iterative scheme to solve nonlinear problems analytically. HAM was introduced at the first time by Liao [1]. Homotopy analysis method has been successfully applied to solving nonlinear algebraic equations [2].

Halley's irrational method is another popular iteration scheme to solve nonlinear equations was proposed by Sir Edmond Halley. To explain the idea of this method suppose that $f(x)=0, f \in C^{2}[a, b]$, by Taylor expanding $f(x)$ around $x_{n}$ discarding higher order terms and setting the result to zero,

$$
f(x)=f\left(x_{n}\right)+f^{\prime}\left(x_{n}\right)\left(x-x_{n}\right)+\frac{1}{2 !} f^{\prime \prime}\left(x_{n}\right)\left(x-x_{n}\right)^{2}+\ldots
$$

now use the first three terms to approximate $f(x)$. That is 


$$
f(x) \approx f\left(x_{n}\right)+f^{\prime}\left(x_{n}\right)\left(x-x_{n}\right)+\frac{1}{2 !} f^{\prime \prime}\left(x_{n}\right)\left(x-x_{n}\right)^{2}
$$

Solving respect to $x-x_{n}$, while setting $f(x)=0$, gives

$$
x-x_{n}=\frac{-f^{\prime}\left(x_{n}\right) \pm \sqrt{f^{\prime 2}\left(x_{n}\right)-2 f\left(x_{n}\right) f^{\prime \prime}\left(x_{n}\right)}}{f^{\prime \prime}\left(x_{n}\right)}
$$

therefore

$$
x_{n+1}=x_{n}+\frac{-f^{\prime}\left(x_{n}\right) \pm \sqrt{f^{\prime 2}\left(x_{n}\right)-2 f\left(x_{n}\right) f^{\prime \prime}\left(x_{n}\right)}}{f^{\prime \prime}\left(x_{n}\right)}
$$

this is Halley's irrational method (Euler) [ 3,4,5,6 ] enjoy third order of convergence. Using Halley's irrational scheme complex and real roots can be founded for a given nonlinear algebraic equation. Halley's irrational method yields a special case of Laguerre's method [7] sometimes attributed to Cauchy [8].

\section{Basic Idea of HAM}

This section discuses the detail of solving nonlinear algebraic equations by using homotopy analysis method.

In particular to illustrate this idea clearly, we need to consider the nonlinear algebraic equation

$$
f(x)=0
$$

Let $\alpha$ be a simple root of it and $f \in C^{3}$ function in the neighborhood of $\alpha$, and suppose that $\left|f^{\prime}(\alpha)\right|>0$.

Because of $f(x)$ has the continuous derivative up to the third order, then this function can be expand in Taylor Expansion around $x$ as following

$$
f(x-\delta)=f(x)-\delta f^{\prime}(x)+f^{\prime \prime}(x) \frac{\delta^{2}}{2 !}+O\left(\delta^{3}\right)
$$

when $x-\delta$ close enough to $\alpha$, equation (2) can be expanded as

$$
f(x-\delta)=0 \approx f(x)-\delta f^{\prime}(x)+f^{\prime \prime}(x) \frac{\delta^{2}}{2 !}
$$

Then solving of $\delta$ gives 


$$
\begin{aligned}
& \delta=\frac{f(x)}{f^{\prime}(x)}+\frac{\delta^{2}}{2 !} \frac{f^{\prime \prime}(x)}{f^{\prime}(x)} \\
& \frac{f(x)}{f^{\prime}(x)}=\delta-\frac{\delta^{2}}{2 !} \frac{f^{\prime \prime}(x)}{f^{\prime}(x)}
\end{aligned}
$$

Based on this relation we define a function

$$
A(\delta)=\delta+\delta^{2} \gamma=c
$$

where

$$
c=\frac{f(x)}{f^{\prime}(x)} \quad \gamma=-\frac{1}{2} \frac{f^{\prime \prime}(x)}{f^{\prime}(x)}
$$

Now we use the idea of homotopy analysis method to solve the equation (1). Using the homotopy parameter $p \in[0,1]$, we construct the so called zero-order deformation equation

$$
(1-p) L\left[\phi(p)-\delta_{0}\right]=p \hbar H(\delta)\{A[\phi(p)]-c\}
$$

Where $\hbar$ a non-zero auxiliary parameter, $L$ an auxiliary linear operator, $H(\delta)$ is a non-zero auxiliary function, $\phi(p)$ is an unknown function, and $\delta_{0}$ is the initial approximation of $\delta$, obviously, when $p=0$ and $p=1$, we have from (3)

$$
L\left[\phi(0)-\delta_{0}\right]=0 \quad \text { and } \quad\{A[\phi(1)]-c\}=0
$$

since $\hbar \neq 0$ and $H(\delta) \neq 0$.

Thus from (3), as $p$ increases from 0 to 1 then the solution $\phi(p)$ increases from $\delta_{0}$ to the solution $\delta$ respectively. So, as $p$ increase from 0 to 1 , equation (3) varies from

$$
L\left[\phi(0)-\delta_{0}\right]=0 \quad \text { to } \quad\{A[\phi(1)]-c\}=0
$$

In topology this is called deformation, and $L\left[\phi(p)-\delta_{0}\right.$ and $A[\phi(p)]-c$ are said to be homotopic.

So that, $\phi(p)$ can be expanding in the Taylor's series respect to $p$ such that

$$
\phi(p)=\sum_{m=0}^{\infty} \delta_{m} p^{m}
$$

where

$$
\delta_{m}=\left.\frac{1}{m !} \frac{d^{m} \phi(p)}{d p^{m}}\right|_{p=0}
$$

It should be mention that one has the great freedom in choosing the auxiliary linear operator, the initial guess, the auxiliary parameter $\hbar$ and the auxiliary function $H(\delta)$, such that the series (4), Taylor's series respect to $p$, is convergent at $p=1$, one has

$$
\delta=\delta_{0}+\sum_{m=0}^{\infty} \delta_{m}
$$


It was founded by Liao (2003) that the convergence rate of homotopy series (4) was adjusted by $\hbar$, and then the equation (5) is the exact solution of (1), whenever this series is known to be convergent.

Setting $H(\delta) \equiv 1$, we have the high - order deformation equation

$$
L\left[\delta_{m}-\chi_{m} \delta_{m-1}\right]=\hbar R_{m}\left(\vec{\delta}_{m-1}\right)
$$

where

$$
\chi_{m}= \begin{cases}0 & \text { if } m \leq 1 \\ 1 & \text { if } m>1\end{cases}
$$

and

$$
R_{m}\left(\vec{\delta}_{m-1}\right)=\vec{\delta}_{m-1}+\gamma \sum_{i=1}^{m-1} \delta_{i} \delta_{m-i-1}-\left(1-\chi_{m}\right) c
$$

where

$$
\delta_{m-1}=\left.\frac{1}{(m-1) !} \frac{d^{m-1} \phi(p)}{d p^{m-1}}\right|_{p=0}
$$

and

$$
\overrightarrow{\delta_{m}}=\left\{\delta_{0}(x), \delta_{1}(x), \ldots, \delta_{m}(x)\right\} .
$$

The subscript $m$ denoted the $m+1$ th-term approximations of $\delta$.

$$
x_{n+1}=x_{n}-\delta\left(x_{n}\right) \text {. }
$$

When $m \geq 1$ one can compute $\delta_{m}$ such as

$$
\delta_{m}=\left(\chi_{m}+\hbar\right) \delta_{m-1}+\hbar \gamma \sum_{k=0}^{m-1} \delta_{k} \delta_{m-1-k}-\hbar\left(1-\chi_{m}\right) c .
$$

The approximation when $m=0$ given by

$$
\alpha=x-\delta \approx x-\delta_{0}=x-c=x-\frac{f(x)}{f^{\prime}(x)}
$$

which is the Newton - Raphson method.

From the approximation of $\delta_{1}$

$$
\begin{aligned}
\alpha & =x-\delta \approx x-\delta_{0}-\delta_{1} \\
& =x-\frac{f(x)}{f^{\prime}(x)}-\left[\hbar \frac{f(x)}{f^{\prime}(x)}-\hbar \frac{f^{\prime \prime}(x)}{2 f^{\prime}(x)} \frac{f^{2}(x)}{f^{\prime 2}(x)}+\hbar \frac{f(x)}{f^{\prime}(x)}\right] \\
& =x-\frac{f(x)}{f^{\prime}(x)}+\hbar \frac{f^{2}(x) f^{\prime \prime}(x)}{2 f^{\prime 3}(x)}
\end{aligned}
$$

therefore,

$$
x_{n+1}=x_{n}-\frac{f\left(x_{n}\right)}{f^{\prime}\left(x_{n}\right)}+\hbar \frac{f^{2}\left(x_{n}\right) f^{\prime \prime}\left(x_{n}\right)}{2 f^{\prime 3}\left(x_{n}\right)}
$$

by the same process the approximation of $\delta_{2}$ gives that

$$
\alpha=x-\delta \approx x-\delta_{0}-\delta_{1}-\delta_{2}
$$




$$
\begin{gathered}
=x-\frac{f(x)}{f^{\prime}(x)}+(\hbar+2) \hbar \frac{f^{2}(x) f^{\prime \prime}(x)}{2 f^{\prime 3}(x)}-\hbar^{2} \frac{f^{3}(x) f^{\prime \prime 2}(x)}{2 f^{\prime 5}(x)} \\
x_{n+1}=x_{n}-\frac{f\left(x_{n}\right)}{f^{\prime}\left(x_{n}\right)}+(\hbar+2) \hbar \frac{f^{2}\left(x_{n}\right) f^{\prime \prime}\left(x_{n}\right)}{2 f^{\prime 3}\left(x_{n}\right)}-\hbar^{2} \frac{f^{3}\left(x_{n}\right) f^{\prime \prime 2}\left(x_{n}\right)}{2 f^{\prime 5}\left(x_{n}\right)}
\end{gathered}
$$

refer to [1] when $\hbar=-1$ the formula (6) is the same as modified Adomian's decomposition method and modified homotopy perturbation method.

We are going to present our contribution which is determine $\hbar$ and set it as a fixed constant in the equation (6), by using Halley's irrational formula. First, arrange this equation as

$$
x_{n+1}=a_{n}+\hbar_{n} b_{n}+\hbar_{n}^{2} c_{n}
$$

where

$$
\begin{array}{r}
a_{n}=x_{n}-\frac{f\left(x_{n}\right)}{f^{\prime}\left(x_{n}\right)} \quad b_{n}=\frac{f^{2}(x) f^{\prime \prime}(x)}{f^{\prime 3}(x)} \quad \text { and } \\
c_{n}=\frac{f^{2}\left(x_{n}\right) f^{\prime \prime}\left(x_{n}\right)}{2 f^{\prime 3}\left(x_{n}\right)}-\frac{f^{3}\left(x_{n}\right) f^{\prime \prime 2}\left(x_{n}\right)}{2 f^{\prime 5}\left(x_{n}\right)}
\end{array}
$$

Now we use Halley's irrational scheme to determine $\hbar$ such that

$$
\hbar_{n+1}=\hbar_{n}+\frac{-g^{\prime}\left(\hbar_{n}\right) \pm \sqrt{g^{\prime 2}\left(\hbar_{n}\right)-2 g\left(\hbar_{n}\right) g^{\prime \prime}\left(\hbar_{n}\right)}}{g^{\prime \prime}\left(\hbar_{n}\right)} \quad, g^{\prime \prime}\left(\hbar_{n}\right) \neq 0
$$

where

$$
g(\hbar)=f\left(a_{n+1}+\hbar b_{n+1}+\hbar^{2} c_{n+1}\right)=0
$$

Therefore,

$$
\hbar_{0}=\frac{b_{0} f^{\prime}\left(a_{0}\right) \pm \sqrt{\left(b_{0} f^{\prime}\left(a_{0}\right)\right)^{2}-2 f\left(a_{0}\right)\left[2 c_{0} f^{\prime}\left(a_{0}\right)+b_{0} f^{\prime \prime}\left(a_{0}\right)\right]}}{2 c_{0} f^{\prime}\left(a_{0}\right)+b_{0} f^{\prime \prime}\left(a_{0}\right)}
$$

So that $\hbar$ is not arbitrary. i.e. $\hbar$ determined by the formula above.

Behind this idea we point out that this procedure has two important advantages. First, because of the \pm and this gives rise to two possible $\hbar_{n+1}$ 's. Second, the $\sqrt{(\ldots)}$ gives rise to the possibility of a complex $\hbar_{n+1}$.

\section{Convergence Analysis}

Throughout this section we study analysis convergence of the current investigation of Halley-HAM (H-HAM), and analyzing the change on $\hbar$.

\subsection{Convergence Analysis of HAM}

To obtain the convergent of the formula (6) 


$$
x_{n+1}=x_{n}-\frac{f\left(x_{n}\right)}{f^{\prime}\left(x_{n}\right)}+\hbar \frac{f^{2}\left(x_{n}\right) f^{\prime \prime}\left(x_{n}\right)}{f^{\prime 3}\left(x_{n}\right)}+\hbar^{2}\left[\frac{f^{2}\left(x_{n}\right) f^{\prime \prime}\left(x_{n}\right)}{2 f^{\prime 3}\left(x_{n}\right)}-\frac{f^{3}\left(x_{n}\right) f^{\prime \prime 2}\left(x_{n}\right)}{2 f^{\prime 5}\left(x_{n}\right)}\right]
$$

Let $\alpha$ be a simple root of $f$. Consider the iteration function $\psi$ defined by

$$
\psi(x)=x-\frac{f(x)}{f^{\prime}(x)}+\hbar \frac{f^{2}(x) f^{\prime \prime}(x)}{f^{\prime 3}(x)}+\hbar^{2}\left[\frac{f^{2}(x) f^{\prime \prime}(x)}{2 f^{\prime 3}(x)}-\frac{f^{3}(x) f^{\prime \prime 2}(x)}{2 f^{\prime 5}(x)}\right]
$$

Here we will replace $\hbar$ by Halley's irrational method.

By derivative

$$
\begin{aligned}
\psi^{\prime}(x)= & 1-\frac{f^{\prime 2}(x)-f(x) f^{\prime \prime}(x)}{f^{\prime 2}(x)}+\hbar \frac{f^{\prime 3}(x)\left[f^{2}(x) f^{\prime \prime \prime}(x)+2 f(x) f^{\prime}(x) f^{\prime \prime}(x)\right]-3 f^{2}(x) f^{\prime 2}(x) f^{\prime \prime 2}(x)}{\left(f^{\prime 3}(x)\right)^{2}} \\
& +\frac{\hbar^{2}}{2}\left[\frac{f^{\prime 3}(x)\left(f^{2}(x) f^{\prime \prime \prime}(x)+2 f(x) f^{\prime}(x) f^{\prime \prime}(x)\right)-3 f^{2}(x) f^{\prime \prime 2}(x) f^{\prime 2}(x)}{\left(f^{\prime 3}(x)\right)^{2}}-\right. \\
& \left.\frac{f^{\prime 5}(x)\left[2 f^{3}(x) f^{\prime \prime}(x) f^{\prime \prime \prime}(x)+3 f^{2}(x) f^{\prime}(x) f^{\prime \prime 2}(x)\right]-5 f^{3}(x) f^{\prime 4}(x) f^{\prime \prime 3}(x)}{\left(f^{\prime 5}(x)\right)^{2}}\right]
\end{aligned}
$$

Because $f(\alpha)=0, \psi(\alpha)=\alpha$, and $\psi^{\prime}(\alpha)=0$ imply that the iterative process $\psi\left(\alpha_{n}\right)=\alpha_{n+1}$ has at least second order of convergence from the equations above. From the Taylor expansion of $\psi\left(x_{n}\right)$ around $x=\alpha$ we obtain the error $e_{n}=\alpha_{n+1}-\alpha$

$$
x_{n+1}=\psi\left(x_{n}\right)=\psi(\alpha)+\psi^{\prime}(\alpha) e_{n}+\psi^{\prime \prime}(\alpha) \frac{e_{n}^{2}}{2 !}+O\left(e^{3}\right)
$$

since $\psi(\alpha)=\alpha$, and $\psi^{\prime}(\alpha)=0$ then

$$
e_{n+1}=\psi^{\prime}(\alpha) e_{n}+\frac{e_{n}^{2}}{2 !} \psi^{\prime \prime}(\alpha)\left(\alpha+\varepsilon e_{n}\right)
$$

where $\varepsilon \in[0,1]$, if $\left|\psi^{\prime \prime}(x)\right| \leq M$ in the neighborhood of $\alpha$, where $M$ is finite positive number, then we get the error estimation

$$
\left|\alpha_{n+1}-\alpha\right| \leq\left|\alpha_{n}-\alpha\right|^{2} \quad M / 2 \text { ! }
$$

This shows that the second order convergence in the neighborhood of $\alpha$. 


\subsection{Convergence Analysis of $\hbar$}

In order to analyze the convergent characteristics of the iteration scheme

$$
\hbar_{n+1}=\hbar_{n}+\frac{-g^{\prime}\left(\hbar_{n}\right) \pm \sqrt{g^{\prime 2}\left(\hbar_{n}\right)-2 g\left(\hbar_{n}\right) g^{\prime \prime}\left(\hbar_{n}\right)}}{g^{\prime \prime}\left(\hbar_{n}\right)}
$$

Let $g(\alpha)=0$, and consider the following iteration function

then, we obtain that:

$$
\phi(\hbar)=\hbar+\frac{-g^{\prime}(\hbar) \pm \sqrt{g^{\prime 2}(\hbar)-2 g(\hbar) g^{\prime \prime}(\hbar)}}{g^{\prime \prime}(\hbar)}
$$

$$
\phi^{\prime}(\hbar)=\frac{\mp g(\hbar) g^{\prime \prime \prime}(\hbar)}{g^{\prime \prime}(\hbar) \sqrt{g^{\prime 2}(\hbar)-2 g(\hbar) g^{\prime \prime}(\hbar)}}+\frac{g^{\prime \prime \prime}(\hbar)\left[g^{\prime}(\hbar) \mp \sqrt{g^{\prime 2}(\hbar)-2 g(\hbar) g^{\prime \prime}(\hbar)}\right]}{g^{\prime \prime 2}(\hbar)}
$$

and

$$
\begin{gathered}
\phi^{\prime \prime}(\hbar)=\frac{g^{\prime} g^{\prime \prime \prime}+g g^{(4)}}{g^{\prime \prime}} \frac{\mp 1}{\sqrt{g^{\prime 2}-2 g g^{\prime \prime}}}+\frac{g g^{\prime \prime \prime 2}}{g^{\prime 2}} \frac{ \pm 1}{\sqrt{g^{\prime 2}-2 g g^{\prime \prime}}}+\frac{\mp g^{2} g^{\prime \prime \prime 2}\left[g^{\prime 2}-2 g g^{\prime \prime}\right]^{\frac{-3}{2}}}{g^{\prime \prime}} \\
\frac{g^{\prime \prime \prime}}{g^{\prime \prime 2}}\left[g^{\prime \prime} \pm \frac{g g^{\prime \prime}}{\sqrt{g^{\prime 2}-2 g g^{\prime \prime}}}\right]+\left[g^{\prime} \mp \sqrt{g^{\prime 2}-2 g g^{\prime \prime}}\right]\left[\frac{g^{(4)}}{g^{\prime \prime 2}}-\frac{2 g^{\prime \prime} g^{\prime \prime \prime 2}}{g^{\prime \prime 4}}\right]
\end{gathered}
$$

since $g(\alpha)=0$ then:

consequently

$$
\begin{aligned}
\phi(\alpha) & =\alpha+\frac{-g^{\prime}(\alpha) \pm\left|g^{\prime}(\alpha)\right|}{g^{\prime \prime}(\alpha)} \\
\phi^{\prime}(\alpha) & =\frac{g^{\prime \prime \prime}(\alpha)\left[g^{\prime}(\alpha) \mp\left|g^{\prime}(\alpha)\right|\right]}{g^{\prime \prime 2}(\alpha)}
\end{aligned}
$$

$$
\phi^{\prime \prime}(\alpha)=\frac{g^{\prime \prime \prime}(\alpha)}{g^{\prime \prime}(\alpha)}\left[1 \mp \frac{g^{\prime}(\alpha)}{\left|g^{\prime}(\alpha)\right|}\right]+\left[g^{\prime}(\alpha) \mp\left|g^{\prime}(\alpha)\right|\right]\left[\frac{g^{(4)}(\alpha)}{g^{\prime \prime 2}(\alpha)}-\frac{2 g^{\prime \prime}(\alpha) g^{\prime \prime \prime 2}(\alpha)}{g^{\prime \prime 4}(\alpha)}\right]
$$

If $g^{\prime}(\alpha) \geq 0$, we take the upper sign and obtain $\phi(\alpha)=\alpha$, and $\phi^{\prime}(\alpha)=\phi^{\prime \prime}(\alpha)=0$. On the other hand, if $g^{\prime}(\alpha)<0$, we take the lower sign and also obtain $\phi(\alpha)=\alpha$ , and $\phi^{\prime}(\alpha)=\phi^{\prime \prime}(\alpha)=0$. It is seen that the root is a fixed point of iteration function $\phi(\hbar) . \quad \phi^{\prime}(\alpha)=\phi^{\prime \prime}(\alpha)=0$ imply that the iterative process $\alpha_{n+1}=\phi\left(\alpha_{n}\right)$ has local cubic convergence. This leads to that for sufficiently smooth iteration function $\phi(\hbar)$ applying the Taylor series expansion around $\hbar=\alpha$, we obtain the error

$$
e_{n+1}=\alpha_{n+1}-\alpha
$$




$$
e_{n+1}=e_{n} \phi^{\prime}(\alpha)+\frac{e_{n}^{2}}{2 !} \phi^{\prime \prime}(\alpha)+\frac{e_{n}^{3}}{3 !} \phi^{\prime \prime \prime}(\alpha)\left(\alpha+\varepsilon e_{n}\right)
$$

Where $\varepsilon \in[0,1]$, since $\phi^{\prime}(\alpha)=\phi^{\prime \prime}(\alpha)=0$ if there is an upper bounded for the third derivative $\left|\phi^{\prime \prime \prime}(\alpha)\right| \leq K$ in the neighborhood of $\alpha$, where $K$ is a finite positive number, the error estimation becomes :

$$
\left|\alpha_{n+1}-\alpha\right| \leq\left|\alpha_{n}-\alpha\right|^{3} M / 3 \text { ! }
$$

Which indicates the local cubic convergence in the neighborhood of $\alpha$.

From both subsections (3.1 and 3.2) one series has second order of convergence and another has 3rd order of convergence respectively. Hence the total is second order of convergence for the equation (1) which can be conclude in next theorem

Theorem 3.2.1. Let $\alpha \in[a, b]$ be a simple zero of a differentiable function $f \in C^{3}[a, b]$. For $\mathrm{h}$ given by

$$
\hbar_{n+1}=\hbar_{n}+\frac{-g^{\prime}\left(\hbar_{n}\right) \pm \sqrt{g^{\prime 2}\left(\hbar_{n}\right)-2 g\left(\hbar_{n}\right) g^{\prime \prime}\left(\hbar_{n}\right)}}{g^{\prime \prime}\left(\hbar_{n}\right)} \quad, g^{\prime \prime}\left(\hbar_{n}\right) \neq 0
$$

then the method defined by

$$
x_{n+1}=x_{n}-\frac{f\left(x_{n}\right)}{f^{\prime}\left(x_{n}\right)}+\hbar \frac{f^{2}\left(x_{n}\right) f^{\prime \prime}\left(x_{n}\right)}{f^{\prime 3}\left(x_{n}\right)}+\hbar^{2}\left[\frac{f^{2}\left(x_{n}\right) f^{\prime \prime}\left(x_{n}\right)}{2 f^{\prime 3}\left(x_{n}\right)}-\frac{f^{3}\left(x_{n}\right) f^{\prime \prime 2}\left(x_{n}\right)}{2 f^{\prime 5}\left(x_{n}\right)}\right]
$$

has second order of convergence.

\section{Numerical Results}

We present some numerical test results for the present contribution Halley irrational-HAM (H-HAM) iterative method to solve some nonlinear algebraic equations. In order to determine the great deal of H-HAM we compare this new method with well-known Newton's method, Halley's irrational method, NewtonHAM, and HAM. 
A. M. Shloof, M. S. Noorani

Table 1: Comparison of the iteration number of some examples

\begin{tabular}{|c|c|c|c|c|c|c|c|}
\hline$f(x)$ & $x_{0}$ & $\mathrm{~N}$ & \multicolumn{2}{|c|}{$\begin{array}{l}\text { Halley } \\
+\end{array}$} & N-HAM & & $\begin{array}{l}\text { HAM } \\
-\end{array}$ \\
\hline $\cos (x)-x e^{x}+x^{2}$ & 0 & 13 & 5 & 3 & 3 & 3 & 3 \\
\hline $\ln (x)$ & 0.5 & 5 & 4 & Div & 4 & 4 & 3 \\
\hline $4 x^{4}-4 x^{2}$ & $-\sqrt{21} / 7$ & 17 & 6 & 4 & 6 & 4 & 6 \\
\hline$x^{3}-e^{-x}$ & 0 & 6 & 4 & 5 & 3 & 31 & 2 \\
\hline $\cos (x)-x$ & 2 & 4 & 8 & 4 & 3 & 2 & 3 \\
\hline$x-2-e^{-x}$ & -10 & 15 & 14 & 12 & 8 & 6 & 6 \\
\hline $\sin ^{2}(x)-x^{2}+1$ & 1 & 5 & 34 & 3 & 3 & 3 & 3 \\
\hline $\sin ^{2}(x)-x^{2}+1$ & -3 & 5 & 3 & 82 & 6 & 3 & 4 \\
\hline
\end{tabular}

Table 2: Examples to show that the efficiency of H-HAM on finding complex roots

\begin{tabular}{|l|l|cc|}
\hline$f(x)$ & $x_{0}$ & H-HAM $(+)$ & H-HAM (-) \\
& & \multicolumn{2}{|c|}{ Root } \\
& & $(5)$ & $(3)$ \\
$x^{3}-e^{-x}$ & -0.5 & $-0.1846255308+1.047326348 \mathrm{i}$ & 0.7728829592 \\
& & $(5)$ & $(5)$ \\
\hline$x-2-e^{x}$ & -10 & $0.860978066-2.073184955 \mathrm{i}$ & $0.8609780865-2.073184157 \mathrm{i}$ \\
\hline
\end{tabular}




\begin{tabular}{|l|l|cc|}
\hline$\left(x^{2}+4\right) \times$ & & $(6)$ & $(5)$ \\
$\left(x^{2}-4 x+5\right)$ & -1.75 & $-2 \mathrm{i}$ & $9 \times 10^{-17}+2 \mathrm{i}$ \\
\hline$(x+3)\left(x^{2}-1\right)$ & & $(3)$ & $(9)$ \\
$\left(x^{2}-4 x+5\right) \times$ & 1.75 & 1 & $2+\mathrm{i}$ \\
$\left(x^{2}+4\right) \times$ & & & \\
$\left(x^{2}+4 x+5\right)$ & & & $(3)$ \\
\hline$x-e^{x}$ & & $(4)$ & $0.3181315057-1.337235702 \mathrm{i}$ \\
\hline
\end{tabular}

\section{Conclusion}

In this paper, based on the homotopy analysis method (HAM), H-HAM has proposed as a new technique among others to solve nonlinear algebraic equations. This method provides us with a simple way to determine $\hbar$. In addition, it is a different manner to find the complex and real roots of a given function. In many cases, because this method contains two iteration directions, this allows us to have two solutions for a specific nonlinear algebraic equation which has multiple roots by giving one initial guess. Finally, refer to table 1 it could be simply conclude that the H-HAM is more efficient than the famous schemes in the literature such as Newton's method, HAM, and Halley's irrational scheme.

\section{References}

[1] S.J. Liao, Beyond perturbation: Introduction to homotopy analsis method, Chapman and Hall/ CRC Press, Boca Raton 2003.

[2] S. Abbasbandy, S.J. Liao, Y. Tan, Newton-Homotopy Analysis Method For Nonlinear Equations, Applied Mathematics and Computation 188 (2007) 1794-1800.

[3] W. Gander, On Halley's Iteration Method, Mathematical Association of America 92 (2) ( 1985) 131-134.

[4] A. Melman, Geometry And Convergence Of Euler's And Halley's Methods, SIAM REV 39 (4) ( 1997) 728-735.

[5] T.R. Scavo, J.B. Thoo, On the geometry of Halley's method, The American mathematical monthly 5(1995) 417-426.

[6] J.F. Traub, Iterative Methods For The Solution Of Equations, Prentice-Hall, Englewood, Cliffs, New Jersey 1964.

[7] S. Hansen, M. Patrick, A Family Of Root Finding Methods, Numerical mathematics 27 (1977) 257-269. 
[8] D.B. Popvski, A Family of One-Point Iteration Formulae For Finding Roots, International journal computation mathematics 8 (1980) 85-88. 